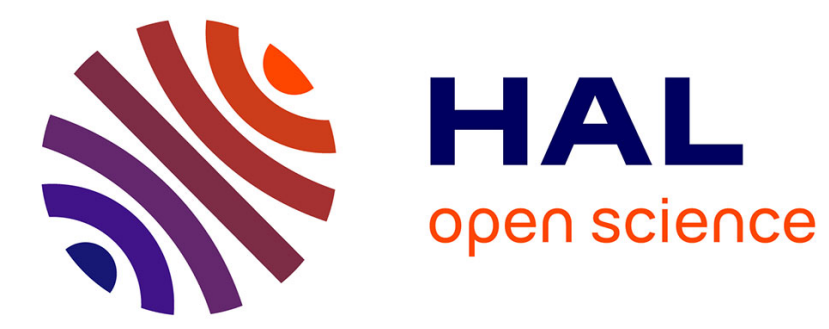

\title{
Les chemins de la mixité conjugale
}

Laura Odasso

\section{To cite this version:}

Laura Odasso. Les chemins de la mixité conjugale. Migrations Société, 2013, 25 (147-148), pp.11-31. hal-01429384

\section{HAL Id: hal-01429384 \\ https://hal.science/hal-01429384}

Submitted on 30 Nov 2017

HAL is a multi-disciplinary open access archive for the deposit and dissemination of scientific research documents, whether they are published or not. The documents may come from teaching and research institutions in France or abroad, or from public or private research centers.
L'archive ouverte pluridisciplinaire HAL, est destinée au dépôt et à la diffusion de documents scientifiques de niveau recherche, publiés ou non, émanant des établissements d'enseignement et de recherche français ou étrangers, des laboratoires publics ou privés. 


\section{LES CHEMINS DE LA MIXITÉ CONJUGALE}

Laura Odasso

Centre d'information et d'études sur les migrations internationales | «Migrations Société »

2013/3 No 147-148 | pages 11 à 32

ISSN 0995-7367

Article disponible en ligne à l'adresse :

https://www.cairn.info/revue-migrations-societe-2013-3-page-11.htm

\section{Pour citer cet article :}

Laura Odasso, «Les chemins de la mixité conjugale 》, Migrations Société 2013/3 ( $\left.\mathrm{N}^{\circ} 147-148\right)$, p. 11-32.

Distribution électronique Cairn.info pour Centre d'information et d'études sur les migrations internationales.

(C) Centre d'information et d'études sur les migrations internationales. Tous droits réservés pour tous pays.

La reproduction ou représentation de cet article, notamment par photocopie, n'est autorisée que dans les limites des conditions générales d'utilisation du site ou, le cas échéant, des conditions générales de la licence souscrite par votre établissement. Toute autre reproduction ou représentation, en tout ou partie, sous quelque forme et de quelque manière que ce soit, est interdite sauf accord préalable et écrit de l'éditeur, en dehors des cas prévus par la législation en vigueur en France. Il est précisé que son stockage dans une base de données est également interdit. 


\section{LES CHEMINS DE LA MIXITÉ CONJUGALE}

Laura ODASSO *

Le présent article puise ses sources dans une enquête socioanthropologique qui examine l'influence du racisme sur les parcours de vie et l'agir des familles dont un partenaire se déclare "arabe". II s'agit d'une recherche comparative entre des couples italo-marocains et italojordaniens vivant en Vénétie (Italie) et des couples franco-marocains et franco-libanais vivant en Alsace (France) ${ }^{2}$.

Les membres de ces familles peuvent être "discrédités " et/ou " discréditables $\|^{3}$ en raison d'une éventuelle spécificité donnée par la présence d'une composante "migrante", "étrangère", "arabe", voire "musulmane", au sein de leur groupe familial. Ces hommes et ces femmes agissent dans un espace en perpétuel changement qui s'alimente de la politisation et de la médiatisation des événements socio-historiques (par exemple, les lois en matière d'immigration, les attentats du $11 \mathrm{sep}$ -

* Docteur en sciences sociales et histoire de l'Afrique du Nord et du Moyen Orient, chercheuse attachée au Laboratoire Cultures et sociétés en Europe (UMR 7236), Université de Strasbourg ; Département d'étude sur l'Asie et sur l'Afrique Méditerranéenne, Université Ca' Foscari, Venise (Italie).

1. Cette appellation requiert des précisions. Nous l'employons selon les critères cumulatifs suivants : “ 1) être de langue et de culture arabes ;2) être ressortissant d'un pays de la Ligue arabe ; enfin 3) se déclarer comme tel ». SCHMIDT DI FRIEDBERG, Ottavia, "La componente araba nell'emigrazione in Italia : elementi per un confronto con altre realtà", in : OPERTI, Laura (a cura di), Cultura araba e società multietnica : per un'educazione interculturale, Torino : Edizioni Bollati Boringhieri, IRRSAE Piemonte, 1998, pp. 93-98 (voir p. 92). Pour une définition de l'"arabité", voir JASSER, Ghaïss, "Voile qui dévoile intégrisme, sexisme et racisme", Nouvelles Questions Féministes, vol. 25, $\mathrm{n}^{\circ}$ 3, 2006, pp. 76-93. Pour une définition d'"être arabe", voir MARDAM-BEY, Farouk ; SANBAR, Elias, Être arabe : entretiens avec Christophe Kantcheff, Arles : Éd. Sindbad-Actes Sud, 2005, 306 p. (cf. p. 9).

Toutes les traductions depuis l'original en italien sont le fait de l'auteur.

2. L'échantillon regroupe des couples représentatifs d'un "double mixte" composé par un partenaire migrant né et ayant vécu jusqu'à l'âge des études secondaires au Maroc, au Liban ou en Jordanie et qui a choisi de partager sa vie avec un partenaire né et élevé en Italie ou en France. Aux récits des membres de 20 familles résidant en Alsace et de 20 familles résidant en Vénétie - conjoints, enfants adolescents et parfois membres de la famille élargie — l'auteur a ajouté des entretiens exploratoires et des entretiens avec des fonctionnaires et des cadres associatifs. Notre enquête a été menée de septembre 2009 à décembre 2011 en Vénétie (provinces de Trévise et Venise) et en Alsace (communauté urbaine de Strasbourg, Mulhouse et alentours de Colmar). En ce qui concerne la notion de "double mixte", voir DELCROIX, Catherine ; GUYAUX, Anne, Double mixte : la rencontre de deux cultures dans le mariage, Paris : Éd. L'Harmattan, 1992, $132 \mathrm{p}$

3. GOFFMAN, Erving, Stigmate : les usages sociaux des handicaps, Paris : Éd. de Minuit, 1989

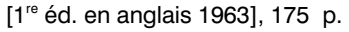


tembre 2001 , etc.), qui représentent leur mixité conjugale ${ }^{4}$ comme étant dérangeante et qui les place a priori dans une situation défavorable. D'une part, ces étrangers font l'objet de représentations homogénéisantes et d'amalgames; d'autre part, les expériences vécues et les contextes de vie sont à l'origine de la manière qu'ils ou elles ont de s'accommoder de façon visible ou silencieuse, individuellement ou collectivement, de cette situation discréditante ou d'y réagir. Leur mixité, en effet, appelle les "l stigmates tribaux $\|^{5}$ qui renvoient à la nationalité, à la religion et à l'origine.

\section{La mixité conjugale : un outil interdisciplinaire}

Dans l'étude de la mixité conjugale les orientations individuelles ne peuvent être saisies sans une analyse de la réorganisation de l'environnement social complexe des individus composant le couple ${ }^{6}$. Le découpage de la notion de mixité montre aussi que si les caractéristiques de sa dimension juridique peuvent se perdre au fil du temps ${ }^{7}$, il en reste un résidu - concernant différentes dimensions sociales - qu'on peut questionner plus en profondeur. II s'agit d'aller au-delà du juridique pour s'aventurer dans une zone de frontières moins visibles attachées aux marques identitaires. Pour réussir leur projet de vie à deux, ces couples cherchent à réagir face au portrait en miroir qui leur est renvoyé de l'extérieur dans un jeu de rôle embrouillé entre les images que ces couples ont d'eux-mêmes et celles qui leur sont renvoyées par la société.

Dans notre travail, la mixité n'est pas conçue comme un objet d'étude autonome, car une réflexion sur les rapports dialectiques entre "altérité" et "commune humanité", dévoile toute l'artificialité du concept

4. Tout le long de l'article nous utiliserons le terme "mixité" comme synonyme de "mixité conjugale".

5. GOFFMAN, Erving, Stigmate : les usages sociaux des handicaps, op. cit.

6. Bien que cette définition date de 1974, elle reste actuelle : « Le sociologue peut considérer comme mariage mixte toute union conjugale conclue entre personnes appartenant à des religions, à des ethnies ou à des races différentes, si ces différences provoquent une réaction de l'environnement social [...]. Qu'est-ce qu'une race, qu'une ethnie, dans le monde occidental contemporain ? ». BENSIMON, Doris ; LAUTMAN, Françoise, "Quelques aspects théoriques de recherche concernant les mariages mixtes", Ethnies, n 4, 1974, pp. 17-40 (voir p. 20).

7. Toute union entre un ressortissant italien ou français et un étranger, quelle que soit son origine, est considérée comme un mariage mixte tant par l'état civil italien que par l'état civil français. Cette mixité peut donc disparaître lorsque le conjoint étranger acquiert la nationalité italienne ou française.

8. La notion se base sur « une forme d'équivalence fondamentale » entre les hommes et les femmes, puisqu'ils et elles “ appartiennent tous au même titre à l'humanité [...] en excluant toutes constructions politiques qui connaissent des esclaves et des sous-hommes ». BOLTANSKI, Luc ; THÉVENOT, Laurent, De la justification : les économies de la grandeur, Paris : Éd. Gallimard, 1991, 483 p. (voir pp. 96 et 163-164). 
de "couple mixte". Cet outil interdisciplinaire qu'est la "mixité conjugale" permet donc d'interroger les enjeux sociétaux qui suivent les "contacts mixtes" au sens plus large 9 . Nous partons du présupposé que ce qui vaut au niveau individuel est valable aussi au niveau collectif et que si la famille est l'unité qui fonde la société, chaque changement sur le plan de la famille peut être un signe révélateur de complexification de la société elle-même.

En outre, la famille est un système vivant qui se développe par étapes successives, selon un "cycle de vie" 10 reliant temps individuel, temps familial - voire social - et temps historique. Cette succession d'étapes s'avère fonctionnelle en vue de saisir les effets de l'expérience migratoire dans les familles considérées dans notre étude. Dans ces configurations, les membres du couple, porteurs d'expériences et de mémoires familiales, négocient constamment leur positionnement et aménagent des stratégies à court et à moyen terme pour faire face aux événements qu'ils subissent ${ }^{11}$. Comme tous les individus, ils participent à d'autres espaces sociaux (école, espace professionnel, milieu associatif, etc.) qui impliquent des ajustements intrafamiliaux et extrafamiliaux qui débordent du cadre familial. L'identité sociale de chaque partenaire apporte dans l'espace amoureux la conscience que l'individu a de sa propre appartenance à des groupes sociaux prédéterminés et les orientations émotionnelles et éthiques que cette appartenance génère.

Le projet familial traduit une dialectique entre intériorité et extériorité, public et privé, et la mixité jalonne ce mouvement avec des contraintes mais aussi avec des ouvertures inattendues ${ }^{12}$. "I La (re)construction des trajectoires [des membres] permet de rendre compte des diverses logiques possibles sous-jacentes à l'élaboration d'un projet $11^{13}$.

L'évolution des familles mixtes est ainsi questionnée par rapport à la migration physique et géographique, c'est-à-dire le déplacement

9. VARRO, Gabrielle, Sociologie de la mixité : de la mixité amoureuse aux mixités sociales et culturelles, Paris : Éd. Belin, 2003, 255 p.

10. Cf. ATTIAS-DONFUT, Claudine ; GALLOU, Rémi ; ROZENKIER, Alain ; WOLF, François-Charles ; DAVEAU, Philippe (sous la direction de), L'enracinement : enquête sur le vieillissement des immigrés en France, Paris : Éd. Armand Colin, 2006, 357 p.

11. Cf. LEGROS-BAWIN, Bernadette, Familles, mariage, divorce : une sociologie des comportements familiaux contemporains, Liège : Éd. Pierre Mardaga, 1988, 213 p.

12. Cf. BERTAUX-WIAME, Isabelle, "Le projet familial", Annales de Vaucresson, $n^{\circ} 1$, Paris : CRIV, 1987, pp. 61-74.

13. LEGROS-BAWIN, Bernadette, Familles, mariage, divorce : une sociologie des comportements familiaux contemporains, op. cit., p. 63. 
d'un état à l'autre, de même que l'évolution " intérieure et de contact " " ${ }^{14}$ de l'Autre. Quels éléments entraînent dans ces couples et familles des réorganisations leur permettant de réussir leur projet familial face aux difficultés?

En interrogeant individuellement une mère, un père et leurs enfants sur leur parcours personnel, sur leur $\|$ mode de vie $\|^{15}$ et sur une série de faits communs, puis en croisant les données recueillies ${ }^{16}$, il est possible de saisir des stratégies dans la durée ainsi que certains éléments qui dépassent les tensions générées par la mixité selon les configurations et les contextes géographiques différents.

\section{Portrait d'un couple italo-jordanien}

Le $\|$ travail biographique $\|^{17}$ réalisé sera présenté ici à partir d'une étude de cas : le parcours d'une famille italo-jordanienne ${ }^{18}$ qui vit dans la communauté urbaine de Venise, en Italie. Paola et Najib sont mariés depuis 1987, ils ont deux filles, Samia et Marinour, âgées respectivement de 18 et 11 ans (elles avaient 16 et 9 ans au moment de notre premier contact avec elles), ils ont vécu à Naples, Rome, Avezzano, Trévise, et enfin, près de Venise. Najib travaille toujours dans la province de Trévise.

14. Dans une perspective sociologique, cette dernière représente l'effort que doit faire le conjoint du pays de résidence pour aller vers l'Autre afin de construire un espace de connaissance, d'échanges et de médiation qui produit des changements concrets au niveau individuel et oblige à "faire l'expérience" du vécu du conjoint en tant qu'étranger, migrant et arabe. II s'agit donc d'un travail biographique qui oblige le partenaire qui n'a jamais migré physiquement à se remettre en question et à se transformer. Cette expérience l'amène à vivre au plus profond de lui-même la situation de l'étranger. Pour les approches psychologiques et psychosociales, voir GOZZOLI, Caterina ; REGALIA, Camillo, Migrazioni e famiglie : percorsi, legami e interventi psicosociali, Milano : Editrice II Mulino, 2005, 253 p. (cf. pp. 13 et 153) ; PANARI, Chiara ; FENAROLI, Patrizia, Famiglie "miste" e identità culturali, Milano : Carocci Editore, 2006, 127 p. (cf. p. 11).

15. Ce concept évoque un processus. L'organisation de la vie quotidienne est liée aux contraintes matérielles et les acteurs sont influencés tant par la sphère productive que par la sphère culturelle et sociale. Voir BERTAUX, Daniel, "Vie quotidienne ou modes de vie ?", Revue Suisse de Sociologie, vol. 9, n 1, mai 1983, dossier "Sociologie de la vie quotidienne et de récits de vie", pp. $67-83$

16. Cf. BERTAUX, Daniel, Les récits de vie : perspective ethnosociologique, Paris : Éd. Nathan, 1997, 127 p. ; DELCROIX, Catherine, "Des récits de vie croisés aux histoires de famille", Current Sociology. Journal of the International sociological association, vol. 43, $\mathrm{n}^{\circ}$ 2-3, 1995, pp. 61-69.

17. INOWLOCKI, Lena ; LUTZ, Helma, "Hard labor : the 'biographical work' of a Turkish migrant woman in Germany. Women in transit between tradition and transformation", European Journal of Women's Studies, vol. 7, $\mathrm{n}^{\circ}$ 3, 2000, special issue, pp. 301-319.

18. Le couple - puis la famille - choisit et affiche un chemin familial dont l'ensemble des membres de la famille se déclare satisfait. II présente des caractéristiques et des stratégies d'action similaires à d'autres cas rencontrés sur le terrain ; bien que " chaque situation soit particulière, les routes à suivre pour atteindre tel ou tel objectif sont en général des routes sociales, communes à bien des acteurs ». BERTAUX-WIAME, Isabelle, "Le projet familial", art. cité, p. 64. 


\section{Encadré I : extrait du récit de vie de Paola}

«Quand je l'ai connu, c'était en 1984, à Naples[...]. Je rentrais à la maison en banlieue, et tu peux imaginer la banlieue napolitaine! Mon frère, qui était venu me chercher pour pas que je rentre seule à la maison, était en train de se moquer de lui en faisant passer des gros mots pour des expressions du dialecte napolitain [...]. J'avais ce keffieh ${ }^{19}$ sur moi, on a échangé des mots et j'ai nommé la poétesse Fadwa Touqan ${ }^{20}$, et lui, il a été frappé ! Il m'a demandé : "Où tu as appris ça? Tu connais sa signification?” Moi j'ai commencé avec mon discours sur les Palestiniens, imprégnée des mobilisations de l'époque, et il m'a dit: "Moi, je suis palestinien!”»

De prime abord, le cas de Paola et Najib semble classique : une mixité juridique initiale, une femme italienne d'éducation chrétienne, un homme jordanien d'origine palestinienne et d'éducation musulmane sunnite. Ils ont grandi dans deux parties du monde distinctes et ont connu une histoire de vie différente, faite cependant d'expériences proches, par la suite réunies en un projet commun.

Paola est née dans la banlieue de Naples, où elle a grandi dans un contexte familial traditionnel : son père, analphabète, ne comprenait pas l'italien, car il ne parlait que le dialecte napolitain, et elle devait lui traduire les informations de la télévision. Un de ses frères, très présent dans sa vie, avait vis-à-vis d'elle un rôle de tuteur, voire de "contrôleur". Le père pensait que ses fils devaient faire des études et que sa fille devait rester à la maison. Cependant, le frère dont elle parle n'avait aucune envie de s'appliquer à réussir à l'école alors qu'elle a dû se battre pour que son père accepte son choix de s'inscrire à l'université pour suivre des études de langues orientales, en arabe et en hébreu. Paola a travaillé pour payer ses études, en faisant, entre autres, du collage d'affiches et du repassage.

De son côté, au moment de leur rencontre, Najib avait un passeport jordanien, mais il était né en Cisjordanie. À l'âge de deux ans il avait quitté sa terre natale suite aux événements de $1967^{21}$ pour s'installer avec sa famille à Zarqa, l'une des villes jordaniennes les plus impor-

19. II s'agit du couvre-chef traditionnel des paysans arabes et des Bédouins, emblème des Palestiniens depuis les années 1960 grâce à sa popularisation par Yasser Arafat. II reste le symbole le plus important des militants pro-palestiniens.

20. Née en 1917 et morte en 2003 , elle est considérée comme l'une des grandes poétesses de la Palestine.

21. En 1967, à l'issue de la guerre des Six Jours, Israël occupe le Sinaï, Gaza, la Cisjordanie, Jérusalem-Est et le plateau du Golan. 
tantes après Amman, composée de quartiers pauvres et d'anciens camps de réfugiés ${ }^{22}$. Issu d'une famille de paysans, sa fratrie est composée de dix frères et sœurs, régie par une mère déterminée qui a fui la Cisjordanie avec ses six premiers enfants, son mari étant à l'époque travailleur migrant au Koweït. Malgré leur histoire familiale mouvementée, le père de Najib rêvait d'offrir à ses enfants la possibilité de faire des études, chance qu'il n'avait pas eve. C'est la raison pour laquelle Najib est parti en Europe, où il a choisi l'Italie pour son coût de la vie plus faible. II y a commencé des études d'architecture, puis, par la suite, il a changé d'orientation et a terminé des études en science du tourisme et marketing à Rome.

\section{- «Marocain ? Mais mon mari est jordano-palestinien!»}

Après leur mariage exogame, les deux partenaires cherchent à maintenir un lien avec leurs familles d'origine respectives, tout en menant leur vie dans un cadre plus indépendant. Ce choix discrédite l'idée selon laquelle les migrants se marieraient ailleurs pour fuir leur famille ou pour rompre avec le passé. Cette réalité peut exister, mais elle est de nature minoritaire ${ }^{23}$, car il semble bien qu'un équilibre entre vie familiale dans le pays d'installation et famille dans le pays d'origine soit recherché. Pour autant, le mariage devient de plus en plus une "affaire privée" qui ne concerne que ses protagonistes.

II n'en demeure pas moins vrai que, au contraire de bien des idées reçues sur ce sujet, les mariages mixtes observés dans notre travail rompent avec des pratiques sociales considérées comme "normales" dans le sens commun, c'est-à-dire les mariages homogames. Ce qui est particulièrement vrai dans l'Italie des années 1980 où le phénomène de l'immigration commençait à prendre forme et, avec lui, celui des mariages binationaux.

22. La ville a été au centre de l'attention internationale en septembre 1970, en raison des dramatiques événements de "Septembre Noir" ; actuellement elle est connue pour être la ville natale d'Abou Moussab al-Zarqaoui, apparemment affilié au groupe islamiste d'Al-Qaïda et responsable de plusieurs attaques terroristes. Voir LAVERGNE, Marc, La Jordanie, Paris : Éd. Khartala, 1996, 249 p. Paola précise que "Zarqa est aussi la ville dans laquelle le frère poliomyélitique de [son] mari a créé une association humanitaire qui s'occupe de l'intégration et de l'insertion linguistique des Pakistanais en Jordanie ».

23. Cf. GOZZOLI, Caterina ; REGALIA, Camillo, Migrazioni e famiglie : percorsi, legami e interventi psicosociali, op. cit., p. 165. 


Encadré2 : extrait du récit de vie de Paola
«Imagine-toi la situation quand je me suis mise avec un étranger il y a 25 ans...
Pour mon père, c'était un choc! Il accordait beaucoup d'importance à mes frères
et moins à moi, donc, quand il a su que je sortais avec un étranger, il m'a
littéralement enfermée à la maison, jusqu'au moment où mon mari est venu chez
nous pour se présenter. Mais avant mon père était allé se renseigner, il avait
demandé des informations au prêtre. Il est rentré à la maison comme un fou et il
m’a dit que j'étais stupide. Il m'a dit : “Un Marocain peut se marier avec 40
femmes ?” Alors moi j'ai dû le convaincre que Najib n'était pas marocain et
qu'il fallait enlever un zéro à la fin : “Non, pas 4o, mais quatre, et seulement
dans certains cas !». De toute façon mon père n'était pas convaincu jusqu'au
moment où il a fait sa connaissance».

Dans cet extrait, comme dans plusieurs récits recueillis pendant l'enquête, nous entendons fréquemment le mot "Marocain" qui, par généralisation, réduit l'ensemble de toutes les nationalités arabes à la seule nationalité marocaine. Cette attitude, justifiée en partie par I'histoire des migrants en Italie, où les Marocains ont été les premiers immigrés arabophones, est représentative de la méconnaissance qui a caractérisé l'Italie, elle-même pays d'émigration. De tels jugements à l'emporte-pièce sont portés tant envers les étrangers qu'envers les Arabes. La réticence à l'égard du mariage "hors du groupe" n'est pas seulement une forme de protection ou de maintien de ce dernier. En Italie, parfois, elle est imprégnée d'ignorance. Comme Paola le souligne : "I Mon père ne disait jamais "arabe" ou "musulman"; à l'époque il parlait de "Marocains". Mon mari est palestinien, mais tu vois Marocain, non? II.

Seuls l'interaction et le partage d'expériences vécues permettent d'aboutir à une restructuration dans le discrédit et à une remise en question, au moins partielle, car individualisée, des idées reçues, comme dans le cas du père de Paola. La famille de Najib en Jordanie fait également preuve de résistance émotive après sa rencontre avec Paola. Comme Najib le souligne : " Quand je leur ai dit ça la première fois au téléphone, c'était difficile, il y a eu presque un refus. Sans connaître la personne, la mentalité, ils partaient sur la base d'un préjugé, mais après, quand ils ont fait sa connaissance, ils ont été contents; au contraire, là, ils l'adorent! II.

L'officialisation du mariage revêt une valeur symbolique pour ces deux familles, italienne et jordanienne, et malgré leur désapprobation 
cette union est finalement acceptée, car institutionnalisée au moyen d'un " processus d'entrée en société " ${ }^{24}$ et légitimée par la signature de l'acte de mariage. En franchissant le passage du célibat à l'union, le couple accepte une norme sociétale de forte valeur dans les deux sociétés de l'époque. Alors que Najib affirme : " Son père m'a dit : "Si tu veux ma fille, il faut que tu l'épouses !" "I, Paola précise : " La première fois qu'on est allé en Jordanie, mon mari a mis longtemps à leur expliquer que nous étions mariés. Les premiers soirs ils n'ont pas voulu nous faire dormir ensemble ; après i'ai été présentée à toute la famille 11.

La figure du père est centrale dans le discours de Paola : il incarne la figure traditionnelle du chef de famille, sa voix pèse dans les choix de sa fille, et cette "tutelle" du patriarche sur sa progéniture incarne bien le type de relation intergénérationnelle familiale. Dans l'affirmation suivante, Paola semble trouver des similitudes entre les dynamiques familiales italiennes et arabes par le biais de l'usage du terme "tutelle"25. Paola le souligne par rapport à l'attitude de son père, qui a "la mentalité d'un "padre padrone"26. La fille passe de sa tutelle à celle d'un autre homme, c'est aussi "pur" Arabe cette mentalité ! Après le mariage, parfois je l'ai retrouvée ! II.

Ce concept est central sous l'angle de la réaction des familles par rapport au choix des filles de se marier avec un musulman ${ }^{27}$. Dans ce récit, la question de la tutelle est le symbole d'une prise de position de cette jeune fille italienne, même s'il est également question des revendications des droits des femmes dans les pays où le statut personnel est inspiré de la charia. Convaincue de sa décision, Paola refuse cette tutelle dans son parcours personnel et familial, mais surtout dans sa migration intérieure dont nous parlerons plus avant. Certains aspects institutionnalisés et structurants dans le monde musulman ne sont-ils pas également présents de manière invisible dans le monde catholique européen?

24. ELIAS, Norbert, La société des individus, Paris : Éd. Fayard, 1991 [1 $1^{\mathrm{re}}$ éd. en allemand 1987], 301 p.

25. En droit musulman, la tutelle est légale, judiciaire ou testamentaire, et elle revient en principe au père. " En cas d'absence [la tutelle] revient, dans l'ordre, au grand-père paternel, au frère germain, au demi-frère consanguin, au neveu germain (fils du frère), au neveu consanguin (fils du demi-frère), à l'oncle, au cousin, etc. selon les règles établies par le statut personnel ». JURISCOPE, "L'autorité parentale en droit libanais", 2002, http://www.juriscope.org/ publications/etudes/autorite-musulman.htm,p. 8 (consulté le 2 juillet 2012).

26. Expression italienne pour désigner un père de famille autoritaire.

27. Souvent sans faire la distinction entre la pratique, la croyance et les habitudes au quotidien et sans tenir compte de l'autodéfinition personnelle des individus. 
On peut observer une tendance au contrôle familial sur les choix matrimoniaux des filles dans les familles françaises et italiennes, protestantes et catholiques interrogées au cours de l'enquête. Le concept de "tutelle", bien que décliné de manière différente entre configurations et contextes, assume une valeur sociologique qui permet d'identifier des similitudes sur la base des rapports de genre. De plus, les deux fiancés acquièrent un statut différent à partir de la célébration du mariage et passent à une autre étape de leur parcours de vie. Ni Paola ni Najib n'avaient envisagé de se marier si vite ni si jeunes ${ }^{28}$, mais en vue de leur émancipation, une fois l'acte de mariage signé à la mairie, ils l'ont assumé et en ont partagé les conséquences. Paola explique : "I'ai rompu avec ma famille et je l'ai suivi. Mon père voulait que je termine ma licence et que je me marie en robe blanche à l'église, le pauvre ! [...]. Mais après mes parents se sont résignés, car nous avions une envie folle de continuer, peut-être parce qu'au-delà du fait que nous sommes des personnes qui venons de pays différents, d'origines différentes, il y a toujours eu l'intention d'être ensemble. Mes parents l'ont compris, et à la fin, maintenant, il y a un grand respect ! II.

Leur projet de vie personnel devient un projet de vie de couple, puis un projet familial. Les deux conjoints attribuent une grande importance à la réussite de leur famille, bien qu'à travers leurs récits transparaissent des conceptions différentes de la famille : la famille européenne, généralement plus individualiste ${ }^{29}$, et la famille proche-orientale, plutôt élargie et communautaire. C'est ainsi que Paola affirme : II Je pense que la famille, ce sont mes filles, mon mari et moi ! Pour lui, il faut tenir compte de toute la famille, celle de Jordanie aussi ! II.

Sans pour autant faire de ces deux modèles un absolu, l'idée que les deux conjoints ont de la famille ne paraît pas coïncider tout à fait. Néanmoins, Paola profite d'une cohabitation élargie avec les frères de son mari pour mieux apprendre l'arabe ${ }^{30}$ : "Un des frères qui a vécu avec nous était utile pour moi [...], lui, il n'a jamais appris l'italien, le pauvre [rires], mais comme on parlait pratiquement toujours en arabe, moi i'ai bien appris l'arabe! II.

28. Paola affirme : “Je pense que ni moi ni lui n'étions convaincus, mais le mariage était le seul moyen de tranquilliser mon père [...]. Peut-être on s'est convaincu par la suite... ".

29. Comme le montre l'enquête, la conception de la famille change selon les récits recueillis. En Italie, elle a une valeur centrale et son unité est importante pour les personnes interviewées, malgré une évolution récente vers la famille mononucléaire et un plus grand individualisme. En revanche, en France, les familles rencontrées, souvent des familles recomposées, semblent être plus autonomes par rapport à leur entourage familial. Le rôle joué par l'Église catholique en Italie est dans cette optique assez important.

30. Cf. GROSJEAN, François, Life with two languages : an introduction to bilingualism, Cambridge : Harvard University Press, 1982, $370 \mathrm{p}$. 
Paola parle la langue de son époux grâce aux bases d'arabe littéraire ${ }^{31}$ étudié à l'université et suite à un voyage en Jordanie, ce qui lui permet de travailler dans cette langue et de se débrouiller sans problème au Moyen-Orient - " en faisant comme toutes les autres II làbas - et d'aider ses filles à en acquérir au moins les bases.

\section{- Une terrona $a^{32}$ et un étranger avec la nationalité italienne}

Après dix ans de mariage, le couple décide d'aller vivre en Vénétie, d'abord à Trévise puis près de Venise.

\section{Encadré 3: extrait du récit de vie de Paola}

«'étais littéralement choquée par l'accueil que j’ai reçu en tant qu'italienne, car la première fois que je suis allée à l'école avec ma fille Samia, ils lui ont dit "terrona!", et c'étaient des Vénitiens. Puis, quand elle a voulu s'inscrire au lycée à Mestre, ça a été une catastrophe, car ils l'ont traitée d'"étrangère!". C'était juste ce que mon mari m'avait dit! Lui, il voulait lui donner un prénom italien, car il me disait: "Tu penses, la pauvre, plus tard elle se sentira étrangère!”. Tu parles! Quelle prophétie, celle de mon mari, et moi, il y a I5 ans, je lui avais répondu: "Non! L'Italie? Mon pays est démocratique, il n'y aura jamais de problèmes de discrimination de ce genre! Mon pays n'est pas raciste!", et au contraire, là, on en a fait l'expérience...»

L'Italie se caractérise par un sens de l'identité nationale faible, les institutions n'ayant pas encore réussi à unifier les parties d'un État jeune, qui a vécu une première phase de migrations internes du Sud vers le Nord. Les pôles industriels, en particulier FIAT à Turin - mais aussi d'autres chantiers et usines - ont attiré les travailleurs du Sud, suscitant alors une première forme de sentiment d'intolérance anti-

31. L'arabe littéraire ou classique est la version la plus "pure" de la langue arabe, qui était aussi la seule à être enseignée dans les universités en Italie jusqu'aux années 2000. Par la suite, en accord avec les changements linguistiques dans le monde arabe, une variante est apparue. Dans certaines situations formelles ainsi que dans les médias de masse, l'arabe littéraire est désormais substitué par l'arabe standard moderne. Les différences entre ces deux types d'arabe sont repérables dans la syntaxe et dans le vocabulaire. En outre, il faut savoir que la Jordanie, comme tous les pays arabes, est marquée par une forte pluriglossie : les enfants apprennent l'arabe (littéraire ou l'arabe standard moderne) à l'école et on ne le parle que dans certaines situations formelles, les variantes dialectales (Palestiniens, dialectes bédouins, etc.) étant utilisées à la maison et dans toutes les situations informelles. L'anglais, la langue étrangère la plus enseignée en Jordanie, est la langue laissée par le colonisateur et est utilisée et mélangée au dialecte par les jeunes et les personnes de classes aisées.

32. Terme péjoratif pour désigner les Italiens du sud de l'Italie dont l'équivalent en français serait "cul-terreux". 
méridionale. La Cassa del Mezzogiorno (Caisse du Midi), organisme public (1950-1992) créé pour promouvoir le développement économique et social du sud de l'Italie, a été souvent prise comme exemple du décalage porteur d'un malaise de plus en plus profond entre le nord et le sud de l'Italie, malaise que la Ligue du Nord a exploité. Un Sud pauvre aidé par l'État et un Nord riche qui se voyait "voler" son argent, voici la synthèse d'un des propos à la base du programme politique de la Ligue du Nord.

Terroni et meridionali sont les appellations méprisantes que les Italiens du Nord utilisent pour désigner les Italiens du Sud, oubliant ainsi le "fondement de l'Unité de l'Italie " ${ }^{33}$. Dans la même logique, le mépris envers les étrangers se développe souvent par le biais d'un étiquetage qui ignore tous les changements survenus au fil du temps. Najib affirme dans son récit : II II y eut des cas de racisme pur, puis des mots violents, en particulier de la part de la Ligue du Nord, car il y a toujours beaucoup d'ignorance. J'espère qu'elle aura des limites II.

Najib a réussi en créant un commerce franchisé dans la restauration rapide de type vente de kebabs et d'autres produits à emporter et il a ouvert des snacks sur tout le territoire italien, principalement dans les provinces de Venise et de Trévise. Bien que citoyen italien depuis 1994, on pouvait encore lire en 2009 , dans des journaux locaux, des articles relatant son expérience et titrant : "L'étranger qui donne du travail aux habitants de la région de Venise $\|^{34}$. Et Paola d'expliquer : "L'ltalie est un pays provincial, à mon avis; au gouvernement nous avons les membres de la Ligue du Nord qui flattent la mentalité provinciale. L'intolérance et le racisme dérivent du fait que l'étranger doit être identifié, il ne peut pas être là, ce n'est pas grave si je ne sais même pas vraiment d'où il vient et quelle langue il parle ! II.

Bien qu'il ait la nationalité italienne depuis des années, Najib reste toujours un étranger pour la presse italienne, comme le souligne Paola : "I C'est un étranger — même s'il est italien! — qui a créé une chaîne franchisée de kebabs qui compte plusieurs magasins, dont certains là où la Ligue est majoritaire, tu comprends ? II. La "citoyenneté invisible" est stratégiquement exploitée par l'homme qui renverse les stigmates en affirmant que "l c'est déjà important que les médias parlent de ça ". C'est pour

33. APRILE, Pino, Terroni : tutto quello che è stato fatto perché gli italiani del Sud diventassero "meridionali", Milano : Piemme Edizioni, 2010, 305 p.

34. "Shockabab. Lo straniero che da lavoro ai veneti : 'Ho vinto la diffidenza'. La storia. II palestinese e il cammello con gli occhiali", Corriere del Veneto du 18-9-2009. 
cette raison que dans les articles où l'on parle de son travail, Najib accepte de "jouer le jeu" des journalistes qui parlent d'intégration à travers la nourriture et qui affirment que "l les jeunes de Trévise aussi aiment le kebab $\|^{35}$. Il se place alors dans une logique de différenciation "nous"/"eux" afin de mettre en lumière sa position d'étranger et de mieux mettre en évidence le pont qu'il a créé : la nourriture, la coordination des travailleurs de différentes nationalités et, surtout, la présence d'une entreprise dirigée par un immigré qui augmente ses profits dans une région qui s'est enrichie grâce à ce que le journaliste et écrivain vénitien Gian Antonio Stella appelle la "logique du travail " interprétée comme la "maladie du travail ". Les provinces de la région étaient caractérisées par la présence de petites et moyennes entreprises locales qui ont depuis toujours attiré et embauché de la main-d'œuvre immigrée ${ }^{36}$.

Najib, en effet, est citoyen italien depuis 15 ans et il a demandé la nationalité italienne afin de pouvoir circuler librement, mais aussi parce qu'il dit appartenir à l'Italie, pays où il vit depuis l'âge de 20 ans et qui lui a offert des possibilités inespérées par rapport à son pays d'origine.

Paola confirme la profonde reconnaissance de son mari à l'égard de l'Italie et, en même temps, elle affirme qu'il reste lié à ses origines. En se souvenant du moment de l'acquisition de la nationalité italienne, elle raconte : " Mon mari a eu la nationalité italienne quand ma première fille est née. Moi i'étais seule avec lui à Rome, mais quand la nationalité lui a été octroyée il m'a dit: "J'ai mon passeport, je pars pour la Palestine !" II y était retourné à huit ans, mais à ce moment son oncle là-bas était en train de mourir. Dix jours après la naissance de notre première fille il est parti pour la Palestine. Ma famille? Ils ont commencé à me faire des ennuis : "Mais quoi ? La naissance de sa première fille ef lui il s'en fout, par envie d'aller d'où il vient? ?". Moi, je l'ai compris ! La petite allait bien, moi j'allais bien et lui il devait aller en Palestine ! "I.

Le cliché de la fuite est récurrent dans les fantasmes sur les partenaires arabes ou musulmans, ce qui est flagrant dans l'expression " aller d'où il vient ". Ces récits des époux offrent un angle d'observation qui permet d'aller plus dans le détail. Paola et Najib s'aménagent

35. "Il Kebab da asporto in franchising piace anche ai giovani trevigiani", Primo Piano, Gazzettino Veneto du 10-11-2009.

36. Cf. STELLA, Gian Antonio, Schei. Dal boom alla rivolta : il mitico Nordest, Milano : Baldini \& Castoldi Editore, 1997, 284 p. 
des espaces d'indépendance, où le rôle du genre et le partage de l'histoire personnelle ont un poids central. La migration intérieure du partenaire autochtone implique une relativisation du regard. Dans le cas de Paola, cette migration s'exprime dans la phrase "I Moi, je l'ai compris ! "1. Cette migration permet d'intégrer au mieux à la vie familiale les parcours identitaires des deux partenaires avec toutes les conséquences qui en dérivent, dont un déracinement qui, dans ce cas, tend vers une gestion positive du transnationalisme. Le fait d'être "ici" et "là-bas" reste l'une des ressources biographiques les plus bénéfiques de ce couple.

Toutefois, Paola remarque un sentiment de fatigue et un changement dans l'attitude de son mari, qui s'est durcie par rapport à l'ltalie. II doit combattre une opinion publique italienne qui stigmatise et craint les migrants, plus particulièrement les musulmans. Selon Paola, "I au bout de 25 ans de vie commune, nous discutons encore des choses importantes, car le couple mixte ne fonctionne pas toujours bien, et en ce moment il ne peut pas bien fonctionner, car il risque de s'affronter sur le terrain historique ! II.

L'un des snacks que Najib avait aidé à ouvrir, géré par un ami jordanien, a été incendié en septembre 2010, et l'ombre du racisme a plané sur cet acte ${ }^{37}$. Marinour, la benjamine de Najib et de Paola, choquée par cette action, a rédigé une lettre - comme sa mère le raconte - qu'elle a mise dans toutes les boîtes aux lettres de l'immeuble où la famille habite sur laquelle on pouvait lire : " Non aux incendies des commerces! Non au racisme ! Je suis une petite fille de neuf ans qui demande seulement un peu de paix. Mon père a été agressé avec un incendie dans son snack et moi j'ai peur ; ma mère m'a expliqué la guerre en Palestine et le racisme, et je ne veux pas que ça se passe aussi en Italie. Pour cela je vous demande de m'aider à ouvrir les portes de la paix. Merci ! II.

Les filles du couple sont actives et réfléchies. Najib et Paola leur ont transmis toutes les compétences et les ressources nécessaires pour résister à la stigmatisation dont elles font l'expérience. Toutefois, la famille est bouleversée par cet acte d'intimidation. Alors que Paola, aigrie, se montre critique : "I Je vous laisse le soin du commentaire, mais si

37. "Kebab al rogo : l'ombra del razzismo. Piromani devastano il locale : 30 mila euro i danni. II titolare, giordano : 'Mai ricevute minacce'”, // Gazzettino di Treviso du 10-9-2010. Parmi les commentaires à l'article publiés en ligne on peut lire plusieurs messages de solidarité, dont, à titre d'exemple : "Solidarité pour ce qui est advenu. J'espère que les associations vont s'unir pour répliquer durement, pour répondre au fait, qu'il soit raciste ou qu'il soit de nature d'intimidation à titre économique ». 
nos enfants doivent vivre avec cette peur, cela signifie que la situation a changé et que les conséquences sont graves! Moi je vois augmenter l'ombre de l'ignorance, il faut essayer ensemble de l'arrêter ! "I, Najib, lui, essaie de maintenir une posture ferme et imperturbable sur ce sujet : "I Je ne ferai rien contre la violence, mais je continuerai à faire beaucoup pour la paix. Il faut se concentrer sur la paix et un jour elle dominera II.

Cet acte démonstratif et violent n'est pas un cas isolé dans la province de Trévise, où la Ligue du Nord s'oppose souvent aux commerces "ethniques". Par exemple, dans le petit village de Vazzola, des membres de ce parti ont demandé le déplacement d'un commerce de kebabs du centre historique vers la banlieue ${ }^{38}$ et ont proposé une discussion pour réglementer la question au conseil municipal.

\section{- I love Palestine !}

La Palestine est présentée par plusieurs interviewés, tant en Italie qu'en France, comme étant une cause qui réunit - au moins à un niveau discursif - tous les interlocuteurs arabophones et des membres de la société civile non nécessairement d'origine arabe ${ }^{39}$. La question de son instrumentalisation a été parfois présentée comme étant un " conflit importé $\|^{40}$ qui s'est souvent retrouvé au cœur des débats des partis de gauche, et des symboles tels que le keffieh, le drapeau palestinien ou Handala, un personnage des caricatures de l'illustrateur Naji al-Ali ${ }^{41}$, apparaissent fréquemment dans les manifestations.

38. "Kebab in centro, forse è meglio in periferia...", // Gazzettino di Treviso du 24-4-2009. L'un des commentaires à l'article conduit à réfléchir sur la forme de phobie à l'égard des étrangers qui se développe en Italie, certains semblant avoir oublié l'histoire même de l'émigration italienne : "Quelle honte ! On devrait faire de même avec les restaurants italiens qui ouvrent dans les autres pays européens. Ensuite on verra si on interdit le risotto aux radis de Trévise en Amérique, comment vous voulez faire?"

39. Cf. SCHMIDT DI FRIEDBERG, Ottavia, "Sentimenti anti-islamici in Italia e in Europa", in : LEANDRO, Annalisa (a cura di), Islam, culture, migrazioni : saggi scelti, Bologna : II Ponte Editoriale, 2007, pp. 104-114.

40. MARDAM-BEY, Farouk ; SANBAR, Elias, Etre arabe : entretiens avec Christophe Kantcheff, op. cit., pp. 282-287.

41. Naji al-Ali, un caricaturiste palestinien dont la famille s'est réfugiée en 1948 dans le camp libanais de Ein-el-Helweh, a travaillé au Koweït pour le journal A/ Qabas. Expulsé de ce pays en 1985, il a vécu à Londres où il fut blessé d'une balle dans la tête le 22 juillet 1987 et succomba à ses blessures un mois plus tard. En 1988, l'Association mondiale des journaux lui attribue le prix Golden Pen of Freedom. L'Association mondiale des journaux le décrit comme l'un des plus importants caricaturistes depuis la fin du XX siècle. Voir l'article "1987 : cartoonist shot in London Street", On this day 1950-2005 BBC, http://news.bbc.co.uk/onthisday/hi/dates/stories/july/ 22/newsid_2516000/2516089.stm 
L'appartenance de Najib au monde palestinien devient un élément de poids pour la réussite de son projet personnel. Si plusieurs mythologies politico-militantes se construisent autour de la question palestinienne, la cause de la lutte contre l'occupation israélienne ${ }^{42}$ est au centre de l'existence de Paola depuis ses études et elle a pris de l'ampleur depuis sa mise en ménage avec Najib. L'“amour" commun de la Palestine, dans le cas spécifique de ce couple, est un signe d'unité militante tant pour le mari que pour la femme, d'autant plus que même leurs enfants expriment un sens fort d'appartenance à cette terre qu'ils revendiquent avec fierté. Récemment la famille a effectué un voyage en Palestine afin que les filles visitent le village natal de leur père. Le voyage a été une expérience forte pour les jeunes, qui connaissent les bases de l'arabe dialectal et qui ont donc une vision plutôt complète de ce qu'elles observent. À ce propos, Samia, la fille aînée de Paola et de Najib, raconte : " Un jour je suis allée à l'école avec le t-shirt "I love Palestine!", et mes camarades à l'école $m$ 'ont dit que je provoquais et $m$ 'ont fait sortir d'une assemblée de classe ! II.

L'expérience vécue sur les lieux d'origine de son père, marqués par les injustices et les souffrances, ainsi que la découverte de la douloureuse mémoire familiale paternelle qui compose aussi sa mémoire familiale ont influencé profondément les dynamiques identitaires de Samia : II Jusqu'en troisième année du collège, je n'avais aucune envie d'être arabe et fille d'un migrant, en plus à moitié. Mes parents m'ont toujours dit que i'étais palestinienne, mais si par hasard quelqu'un me demandait des choses sur mon prénom, je disais que mon père était un peu jordanien et je passais à un autre discours. Depuis le lycée, je fais valoir mon identité arabo-palestinienne : je ne dis pas que je suis un peu, mais que je suis complètement arabe ! II.

Pour la jeune fille, ses origines jordano-palestiniennes sont une source de fierté, un aspect qu'elle souligne souvent quand elle parle avec ses camarades de classe, des partisans de la ligne politique de la Ligue du Nord. Dans les informations qu'elle donne sur elle-même sur sa page Facebook, Samia écrit : « Vous ne devez pas vous tromper à cause de mon prénom à consonance arabe : je ne suis pas une terroriste " ; "l'idéologie, l'idéologie, malgré tout je crois encore qu'elle existe ; c'est la passion, l'obsession de ta diversité qui, pour le moment, où se trouve-t-elle? On ne

42. Paola est engagée dans la lutte contre l'injustice d'une manière générale : son militantisme porte sur plusieurs sujets d'actualité. 
sait pas $11^{43}$. Les enfants, comme les adolescents, sont souvent influencés par leur environnement familial tant de manière positive que négative : II II s'agit de comprendre le racisme comme un phénomène diffus et familier, interne à notre société, intériorisé par chacun de nous, et de travailler sur le terrain de production de ce phénomène $1{ }^{44}$. Samia développe un sens critique vis-à-vis de son pays de résidence et de naissance. Par exemple, souvent, en entendant son nom et son prénom, on lui demande son origine et celle de son père : " D'où vient-il ? ". Actuellement elle n'a plus honte, mais il lui semble absurde que, dans un pays comme l'Italie, cette question soit encore et toujours posée. Elle sourit lorsqu'elle dit : "I Alors que mon père voulait $m$ 'appeler Serena!".

Paola, militante, se positionne par rapport au territoire de résidence et à ses réactions en tant qu'enseignante : pour elle, l'école est un espace de transmission des connaissances en termes critiques et de reconnaissance des identités des enfants qu'elle a dans sa classe. Elle invite sa fille à suivre la même démarche, mais Samia lui fait noter que pour elle c'est plus simple : "I Tu ne peux pas comprendre, car tu n'es pas arabe! Moi, je suis à moitié arabe ! II.

La mère et la fille vivent deux formes de discrimination différentes. Paola s'interroge sur le poids de l'école, espace de socialisation secondaire, important pour les enfants. Elle présente dans son récit des exemples éclairants d'événements quotidiens et de ses réactions, car elle est persuadée qu'll il n'y a pas d'éruption volcanique sans magma souterrain $1{ }^{45}$. Sa force et son action sont représentatives de ces mères qui arrivent à questionner leur positionnement par rapport aux événements en mobilisant des "ressources subjectives ${ }^{46}$ pour se profiler comme des médiatrices : " Ma fille aînée a grandi et aujourd'hui elle est capable de répondre, de réagir, mais je lis sur son visage le même découragement qu'elle avait quatre ans auparavant, une tristesse profonde face à ces choses méchantes. Aussi, même si on ne les a faites que pour rire, elles sont gratuites !

\footnotetext{
43. II s'agit d'un extrait du texte de la chanson Destra Sinistra (2001) de Giorgio Gaber.

44. TABET, Paola, "Comme s'ils avaient la peau juste", ProChoix, n 16, 2000, pp. 19-26.

45. Ibidem, p. 19.

46. "Les ressources objectives accumulées ou héritées, que Bourdieu appelle des "capitaux" économiques, culturels, sociaux et symboliques, ressources auxquelles il faut joindre les ressources de pouvoir liées à l'occupation d'une place d'autorité dans une institution disposant de ressources collectives : les personnes [...] sont en général dépourvues des premières comme des secondes, mais ce ne sont pas là les seules ressources dont disposent les individus. En effet, ils portent en eux des ressources subjectives. Les "compétences pratiques" et "les compétences discursives" définies par Giddens en font par exemple partie ". DELCROIX, Catherine, Ombres et lumières de la famille Nour : comment certains résistent à la précarité ?, Paris : Éd. Payot, 2005, 249 p. (voir p. 227).
} 
Racisme... ma fille, malgré ce qu'elle a vu en Palestine - les soldats, le mur, l'occupation - elle a eu la force de s'indigner et de se rebeller contre des expressions moqueuses envers les juifs 11.

En tout état de cause, force est de souligner que les programmes scolaires sont assez eurocentrés et laissent peu d'espace au relativisme culturel. La dimension étatique est du ressort de la géographie, qui est étudiée d'un seul point de vue ; or la géographie est justement ce qui doit permettre d'avoir un regard informé et comparatif sur le monde. Paola explique : "I Un jour je rentre à la maison et ma benjamine, en CM 1, me dit un peu confuse et énervée que l'enseignant de religion, en expliquant la vie de Jésus, avait dit que la Palestine s'appelat Israël. Ma fille $m$ 'a demandé : "Non, mais alors, quand on a traversé le mur et on est allé chez oncle Zyad, on est allé où ? Ce n'était pas la Palestine ?" Et moi : “C'est clair que c'était la Palestine ! C'est la Palestine !". Je pense aller parler avec l'enseignant de religion, munie d'une grande patience et d'un peu de saine ironie napolitaine, qui dans certains cas m'évite de faire des dégâts 11.

L'expérience de Marinour montre le décalage qui existe entre la transmission familiale et les notions qui lui sont enseignées à l'école. Paola est consciente que son choix de mixité conjugale n'est pas simple. Elle affirme que "l le fait d'épouser un Arabe est accaparant, ef un Palestinien encore plus ; c'est un Arabe en mouvement, en perpétuelle construction. Moi, i'aime le monde arabe, et lui, il aime l'Italie, et je pense que ça c'est indépendamment l'un de l'autre. Peut-être qu'on s'aime car on reconnaît dans l'autre un monde qui nous a fascinés, captivés, et vis-àvis duquel nous nous sommes mis en marche... moi vers le Moyen-Orient et lui vers l'Occident ! II.

Paola peut demander la nationalité jordanienne, Samia et Marinour l'ont déjà ${ }^{47}$ et elles peuvent rester dans le pays d'origine de leur père.

47. L'article 8 du Code de la nationalité jordanien $n^{\circ} 6$ de 1954 stipule qu'« une femme d'origine étrangère qui épouse un citoyen jordanien peut acquérir la nationalité jordanienne, si elle le souhaite, en effectuant une déclaration écrite à cet effet trois ans après son mariage si elle est originaire d'un pays arabe ; cinq ans après son mariage si elle n'est pas originaire d'un pays arabe ». EUROMED, Rapport national d'analyse de la situation : droits humains des femmes et égalité hommes-femmes. Jordanie, Programme Promouvoir l'égalité entre les hommes et les femmes dans la région euro-méditerranéenne (2008-2011), juillet 2010, 47 p., http://www.euromedgenderequality.org/image.php?id=79 


\section{- Conversion rationnelle par voie intellectuelle}

Paola et Najib forment un couple binational, mais ils sont aussi, comme l'observe Paola, un couple musulman, car elle a décidé de se convertir à l'islam pendant ses études : "Mon couple est formé par un Arabe de religion musulmane et par une Italienne. Moi, je me suis convertie en 1987 sur la base de mes études, mais de ce moment-là à maintenant mon mari ne m'a jamais poussée à lire une seule page du Coran. Moi i'éduque mes filles dans cette religion, et si elles en connaissent des choses, c'est à moi qu'elles le doivent. Quand i'ai connu mon mari, il n'était pas une personne religieuse! [...]. Je me sens musulmane, je ne porte pas le voile ici et je me sens comme ça indépendamment du fait que mon mari est, a été ou sera musulman ! [...]. En revanche, je n'accepte pas les femmes italiennes converties qui n'ont pas eu une approche scientifique de l'islam et qui vivent de manière extrême, à tel point que ce sentiment élimine tout leur patrimoine de femme vécu dans un milieu démocratique... parce que c'est là la question, il ne faut pas tourner autour : l'islam est une chose et la société arabo-islamique en est une autre, qui ne donne pas certains droits aux femmes. Dans le Coran tu ne trouveras jamais un mot qui te dit que la femme doit être traitée comme un appareil électroménager ! "I.

Paola se dit autonome en la matière, et il s'agit d'une conversion rationnelle ${ }^{48}$ qu'elle a décidé pendant une période au cours de laquelle les liens de son mari avec la religion s'étaient distendus. Étant jeune immigré à l'époque, il ne respectait pas à la lettre les normes prescrites, c'est-à-dire les cinq piliers de l'islam, et vivait des expériences auxquelles il n'avait pas eu accès auparavant. Najib affirme n'avoir jamais parlé de conversion avec sa femme : " Je ne suis pas trop religieux. Un jour elle m'a dit qu'elle avait décidé de se convertir à l'islam. Et moi : "Mais pourquoi ce choix-là ?" Elle m'a répondu : "J'ai connu ce que je ne connaissais pas !", donc elle a décidé de se convertir 11.

Ainsi, Paola est convaincue que sa conversion est le résultat d'un parcours intellectuel, de ses études en langue arabe, qui lui ont permis d'effectuer une "migration intérieure" profonde. Le voile n'est qu'un symbole : elle ne le porte pas en Italie, mais a décidé de le porter en Jordanie, où la famille de son mari lui donne une valeur centrale : "La famille de mon mari savait que moi j'étais convertie. Pour certains d'entre eux cela n'était pas assez, il fallait porter le voile, et moi je ne

48. Cf. ALLIEVI, Stefano, Les convertis à l'islam : les nouveaux musulmans d'Europe, Paris : Éd. L'Harmattan, 1999, 383 p. (voir p. 123). 
l'ai pas fait pendant longtemps. J'ai commencé à le porter pour être à l'aise. Je me sens mieux avec dans la famille de mon mari, où presque toutes les femmes sont voilées, et quand je suis seule dans la maison à Amman. J'ai mis des années pour comprendre que dans la maison de mon mari le foulard n'avait pas une dimension religieuse, mais plutôt traditionnelle ; je le prends actuellement comme une forme de respect. Je leur ai dit que le voile, je le mets là avec eux, pour me sentir plus proche d'eux, avec leur groupe familial, mais en Italie je ne peux pas le mettre, je n'arrive pas, car cela ne fait pas partie de moi ! II.

Pour Paola, il s'agit de se fondre complètement dans le milieu d'origine de son mari, montrant ainsi une forme de respect qui n'élimine pas ses particularités de femme italienne. Le fait de "faire comme tous les autres $1{ }^{49}$ pourrait être une vision purement eurocentrée, une idée qui s'appliquerait en Europe ; or, dans ce cas, Paola l'applique en Jordanie et en Palestine : l'usage de la langue, la connaissance des règles sociales, l'usage du voile la positionnent au centre d'un monde qu'elle intègre mais qu'elle critique aussi avec respect. Toutefois, elle insiste sur le fait qu'elle reste "italienne", avec ses spécificités, et qu'elle fait des efforts pour sa belle-famille. Et d'affirmer : II Je pense que c'est parce qu'on ne s'est jamais renfermé chacun rigidement derrière son monde, moi derrière mon Occident et lui derrière son Orient. On a toujours cherché à les mélanger. Ça se reflète aussi dans le travail ${ }^{50}$; voilà, pour lui, c'est plus évident, mais moi aussi quand je suis en classe, quand j'enseigne, il faut que i'y mette quelque chose du monde arabe 11 .

À partir de ce récit on comprend que Paola a abandonné son propre territoire pour découvrir celui de son mari, sous l'influence de son parcours préalable à son histoire d'amour.

\section{Chemins possibles}

Le cas extrait de notre enquête que nous avons exposé ici propose des indices de portée générale aussi bien que des spécificités qui poussent à éviter de faire une analyse superficielle de la mixité. Par

49. VARRO, Gabrielle, "Le sourire du chat, ou les temps de l'assimilation", Temporalités, $\mathrm{n}^{\circ} 1$, 2004, http://temporalites.revues.org/578, consulté le 6 juillet 2012.

50. L'activité professionnelle, qui appartient à la sphère publique, a des résonances dans la sphère privée familiale et vice-versa. Pendant ses études, par exemple, Paola a fait des petits boulots pour gagner de l'argent, puis elle a été archiviste auprès d'une télévision satellitaire arabe basée dans la vallée d'Avezzano, au centre-ouest de l'Italie, puis médiatrice familiale et enfin institutrice. Najib a travaillé comme aide-cuisinier à Rome, puis il a géré des restaurants autour de Venise. 
exemple, la particularité de cette famille réside dans une homogamie évidente (milieu social d'origine, âge, religion à la suite de la conversion de Paola, langue, etc.) bien que, avec une analyse attentive, d'autres éléments moins objectivables émergent (connaissance préalable de l'"arabicité", intérêt pour la Palestine, militantisme, amour de Najib pour l'Italie, etc.). En définitive, la mixité de ce couple est moins forte que supposée initialement; en outre, elle a changé avec le temps et la volonté de vivre à deux. En observant les contextes de résidence de la famille, la mixité est un révélateur des frontières mises en place par l'environnement. Ces frontières représentent des défis qui renforcent la volonté du couple de voir aboutir son projet de vie.

La stabilité économique de la famille suggère un exemple de "mode de vie". Les deux conjoints ont abouti dans leur projet professionnel : le mari, après avoir été un étudiant immigré, est devenu un entrepreneur qui offre des produits "mixtes" (italiens et arabes) à un public varié dans un territoire assez hostile aux migrants. Sa femme, satisfaite de ses expériences de travail, assume son double rôle d'épouse et de mère : ses ressources subjectives, importantes dans l'espace privé, deviennent un " capital d'expérience biographique " à utiliser aussi dans le milieu professionnel ${ }^{51}$.

Bien que Paola semble avoir fait la majorité des concessions ${ }^{52}$, il ne s'agit pas de les observer sous l'angle de la perte, mais plutôt sous celui de l'ouverture.

La réussite du projet familial ressort dans la parentalité. La transmission de la mémoire familiale forge le caractère des filles, qui savent d'où elles viennent et regardent le futur avec des ressources identitaires à partir desquelles elles peuvent commencer à construire leur projet personnel. La Palestine, en particulier, constitutive de la figure du père, alimente le militantisme de la mère et rappelle aux filles une partie de leurs racines en tant qu'appartenance et non pas comme simple identité reproduite. Les filles acquièrent ainsi la conscience d'une histoire familiale qu'elles transformeront peut-être à leur tour.

51. DELCROIX, Catherine, "Ressources subjectives et construction d'un capital d'expérience biographique : l'exemple des médiatrices socio-culturelles", in : DARDY, Claudine ; FRÉTIGNÉ, Cédric (coordonné par), L'expérience professionnelle et personnelle en questions, Paris : Éd. L'Harmattan, 2004, pp. 83-116.

52. Cf. COLLET, Beate, "Pluralité contemporaine de la mixité conjugale et position asymétrique entre les sexes", Diasporas. Histoire et sociétés, $\mathrm{n}^{\circ}$ 15, avril 2010, pp. 53-67. 


\section{En guise de conclusion}

Pour revenir sur le rapport entre mixité et projet familial, la valeur de la double migration (géographique ou physique et intérieure ou de contact) et de la mémoire familiale semble primordiale. Bien que cette dernière soit une reconstruction d'une histoire qui aide les individus à " se donner du sens $\|^{53}$, il s'agit d'un travail créatif qui émerge dans les étapes du cycle de vie et dans les choix au quotidien grâce à la dialectique entre le temps historique, le temps biographique, le contexte socioculturel et le vécu subjectif.

La négociation et le bricolage quotidiens placent les familles mixtes dans un " entre-deux $"{ }^{54}$, un espace innovateur qu'il faut valoriser ; c'est dans cette dimension liminaire que les éléments mobilisés pour analyser l'entretien se mélangent et confirment la validité de la "mixité conjugale" comme clé de lecture des interactions sociales en général. Les stigmates tribaux et les rapports de genre, classe, génération créent des catégories nouvelles qui perdent les définitions statiques en devenant des options individuelles auxquelles les parcours des membres qui composent les familles mixtes confrontent le chercheur.

La mixité est donc une catégorie utile à la reconnaissance de la pluralité des appartenances ${ }^{55}$ qui aide à déconstruire des préjugés dangereux, "l car c'est notre regard qui enferme souvent les autres dans leurs plus étroites appartenances, et c'est notre regard aussi qui peut les libérer $\|^{56}$. Afin d'éviter toute généralisation, l'analyse dans la durée de la " puissance d'agir $\|^{57}$, de la transmission de la mémoire familiale et de la consolidation des ressources subjectives, voire d'un capital d'expérience biographique, doit être articulée au contexte géographique et aux événements historiques.

53. MUXEL, Anne, Individu et mémoire familiale, Paris : Éd. Nathan, 1996, 226 p. (voir p. 9).

54. BHABHA, Homy, The location of culture, London : Routledge Publishers, 1994, 285 p.

55. Cf. VARRO, Gabrielle, "Mettre la 'mixité' à la place de l'origine' ?", in : COLLET, Beate ; PHILIPPE, Claudine (sous la direction de), Mixités : variations autour d'une notion transversale, Paris : Éd. L'Harmattan, 2008, pp. 201-218.

56. MAALOUF, Amin, Les identités meurtrières, Paris : Éd. Grasset, 1998, 210 p. (voir p. 32).

57. La puissance d'agir en vue de l'émancipation montre toute l'importance de la créativité humaine malgré les effets de la domination. L'émancipation « détermine la façon dont les hommes et les femmes se représentent, construisent et gèrent les relations qu'ils entretiennent les uns avec les autres et plus largement leurs rapports à l'espace social public et privé ». LE QUENTREC, Yannick, "Myriam la militante : entre domination et puissance d'agir", in : CARDON, Pierre ; PFEFFERKORN, Roland ; KERGOAT, Danièle (sous la direction de), Chemins de l'émancipation et rapports sociaux de sexe, Paris : Éd. La Dispute, 2009, pp. 219-234 (voir pp. 219-220). 